\title{
p-Hydroxybenzoic acid ( $p$-HA) modified polymeric micelles for brain-targeted docetaxel delivery
}

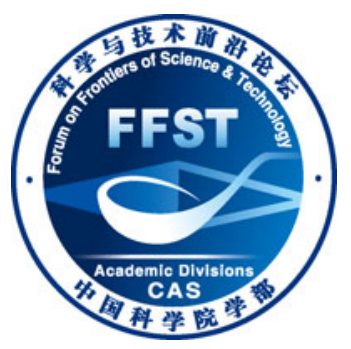

\author{
ZHANG ZhiXin ${ }^{1,2}$, WEI XiaoLi ${ }^{1,2}$, ZHANG XiaoYu $^{1,2} \&$ LU WeiYue ${ }^{1,2,3^{*}}$ \\ ${ }^{1}$ Department of Pharmaceutics, School of Pharmacy, Fudan University, Shanghai 201203, China; \\ ${ }^{2}$ Key Laboratory of Smart Drug Delivery (Fudan University), Ministry of Education \& The Chinese People's Liberation Army, \\ Shanghai 201203, China; \\ ${ }^{3}$ State Key Laboratory of Molecular Engineering of Polymers (Fudan University), Shanghai 201203, China
}

Received July 14, 2012; accepted September 19, 2012; published online April 10, 2013

\begin{abstract}
Chemotherapies for brain diseases have been hampered due to the inability of transport of drug across the blood-brain barrier (BBB). In order to overcome the barrier, $p$-hydroxybenzoic acid ( $p$-HA), a small molecule of benzamide analogue, was used as a ligand for brain-targeted drug delivery. The $p$-HA was conjugated to PEG-DSPE to form $p$-HA-PEG-DSPE. Docetaxel-loaded polymeric micelles were prepared by a thin-film hydration method using methoxy-poly(ethylene glycol)-distearoylphosphatidylethanolamine ( $\mathrm{mPEG}_{2000}$-DSPE) as a carrier and the $p$-HA-PEG-DSPE as a brain targeted material. The prepared micelles showed spherical with a mean diameter of $(18 \pm 3) \mathrm{nm}$. Encapsulation efficiency and drug loading were $(83.49 \pm 1.3) \%,(7.7 \pm 1.2) \%$ for unmodified micelles and $(80.65 \pm 1.6) \%,(7.47 \pm 1.8) \%$ for $p$-HA-modified micelles, respectively. In vitro cellular uptake experiments showed that the $p$-HA-modified micelles increased BCECs cellular uptake by 1.2 times compared to the unmodified micelles. Ex vivo near-infrared fluorescence imaging showed that brain uptake of the $p$-HA-modified micelles was 1.3-1.8 times higher than that of the unmodified micelles. In vitro cytotoxicity assay against glioblastoma cell U87 MG showed that inhibition rate of the $p$-HA-modified micelles increased by 1.2 times compared to that of the unmodified micelles and 1.7 times compared to that of DTX. Survival time of nude mice bearing intracranial glioblastoma showed that the lifetime of saline group, Taxotere group, mPEG-DSPE/DTX micelles group and $p$-HA-PEG-DSPE/DTX micelles group was 22, 27, 32 and $45.8 \mathrm{~d}$, representively, which indicated that anti-glioblastoma activity of DTX could be significantly enhanced by the $p$-HA-modified polymeric micelles. These results demonstrated that the $p$-HA-modified micelles could be a promising brain-targeted drug delivery system for hydrophobic drugs against glioblastoma.
\end{abstract}

p-hydroxybenzoic acid ( $p$-HA), brain-targeting, polymeric micelles, docetaxel, glioblastoma

Citation: $\quad$ Zhang Z X, Wei X L, Zhang X Y, et al. $p$-Hydroxybenzoic acid ( $p$-HA) modified polymeric micelles for brain-targeted docetaxel delivery. Chin Sci Bull, 2013, 58: 2651-2656, doi: 10.1007/s11434-013-5760-z

Blood-brain barrier (BBB) is a physical dynamic barrier between brain and blood, which serves as a protection barrier to effectively prevent certain foreign substances from entering the brain blood circulation. Due to poor permeability of the BBB, nearly $100 \%$ of large molecules and $98 \%$ of small molecules are forbidden access to central nervous system (CNS). Consequently, it hampers treatment and diagnosis of CNS diseases such as Alzheimer's diseases, brain tumors, and Parkinson's diseases [1-4].

*Corresponding author (email: wylu@ @ shmu.edu.cn)
To deliver therapeutic agents across the BBB, mechanisms for transport of endogenous molecules into the brain have been employed such as receptor-mediated endocytosis, adsorptive mediated transcytosis, transporter uptake and membrane permeation of lipophilic molecules $[5,6]$. Receptor-mediated drug delivery systems represent very promising non-invasive strategy for active targeted drug delivery to the site of brain tumors [7-9]. Various receptors and transporters are present on the luminal endothelial plasma membranes, including the transferrin receptor [10], the insulin receptor [11], endothelial growth factor receptor 
[12], low-density lipoprotein receptor [13] and glucose transporter [14] providing binding targets for drug delivery systems.

Benzamide analogue is a kind of small molecules used for the treatment of central nervous diseases. Among them, tiapride could be used for the treatment of behavioral disturbances and non-cognitive symptoms in children agitation; sultopride has strong antipsychotic effects $[15,16]$. Benzamide analogues were found to have high affinity with dopamine receptors that are prominent in most parts of CNS. D1 and D2 dopamine receptors could be found in nigrostriatal pathway comprising the neurons of the substantia nigra and neostriatum [17]. In recent years, a considerable amount of research has been directed towards synthesis of substituted benzamides as ligands for visualization of dopamine D2 receptor binding in the brain by positron emission tomography [18].

Of the nanopartical drug delivery systems, PEG-lipid micelles have been extensively used as vehicles for hydrophobic drugs due to the high stability, controlled drug release, and low toxicity [19-24]. In the present study, a novel brain-targeted nanoparticle drug delivery system based on a small molecule ligand ( $p$-Hydroxybenzoic Acid, $p$-HA) was developed [25]. Few studies have reported on PEG-DSPE micelles modified with $p$-HA. In this study, $p$-HA-modified micelles was first established, then in vitro and in vivo targeting effect of the micelles was investigated. The anti-gliobalstoma efficacy of DTX-loaded micelles was studied on intracranial glioblastoma bearing nude mice.

\section{Materials and methods}

\subsection{Materials}

DIEA (Diisopropylethylamine) were supplied by Fluka (Milwaukee, USA). Docetaxel was kindly provided by Prof. Y. P. Li from Shanghai Institute of Materia Medica (SIMM). EDC $\cdot \mathrm{HCl}$ (1-Ethyl-3-(3-dimethyllaminopropyl) carbodiimide hydrochloride) were purchased from GL Biochem Ltd (Shanghai, China). mPEG 2000 -DSPE (methoxy-poly(ethylene glycol)-distearoylphosphatidylethanolamine) was purchased from Lipoid GmbH (Ludwigshafen, Germany). $\mathrm{NH}_{2}-$ PEG-DSPE was purchased from Laysan Bio Co. (USA). DiR (1,1'-dioctadecyl-3,3,3',3'tetramethyl indotricarbocyanine iodide) was from Invitrogen (USA). DMEM medium and FBS (fetal bovine serum) were purchased from Gibco Co. (USA). Methanol, acetonitrile, and other HPLC grade reagents were obtained from Fisher Scientific (USA). All the chemicals were analytic reagent grade.

\subsection{Cell lines and animals}

Brain capillary endothelial cells (BCECs) were kindly provided by Prof. X. G. Jiang (School of Pharmacy, Fudan University). U87 MG cell line was obtained from the Insti- tute of Biochemistry and Cell Biology, Shanghai Institute for Biological Sciences, Chinese Academy of Sciences. Both cells were cultured in DMEM medium, supplemented with $10 \% \mathrm{FBS}, 100 \mathrm{IU} / \mathrm{mL}$ penicillin and $100 \mathrm{mg} / \mathrm{mL}$ streptomycin sulfate. All the cells were cultured in incubators maintained at $37^{\circ} \mathrm{C}$ with $5 \% \mathrm{CO}_{2}$ under fully humidified conditions. All the experiments were performed on cells (passages from 5 to 30 ) in the logarithmic phase of growth.

$\mathrm{BALB} / \mathrm{c}$ nude mice (male, 4-5 weeks, weighing 18-20 g) were supplied by Department of Experimental Animals, Fudan University (Shanghai, China). All the animal experiments were carried out in accordance with guidelines evaluated and approved by the Ethics Committee of Fudan University.

\subsection{Synthesis and characterization of $p$-HA-PEG- DSPE}

p-HA (27.6 mg) was dissolved in anhydrous DMF (3 mL) together with NHS and EDC. $\mathrm{HCl}$. Then $20 \mu \mathrm{L}$ of DIEA were added and incubated for $3 \mathrm{~h}$. The resulting mixture was centrifuged and the supernatant was added dropwise into a solution of $\mathrm{NH}_{2}$-PEG-DSPE in anhydrous DMF (72 $\mathrm{mg} / \mathrm{mL}$ ). The solution was stirred overnight at room temperature and precipitated using cold diethyl ether for three times. The precipitation was then vacuum-dried for $2 \mathrm{~h}$. The resulting $p$-HA-PEG-DSPE was purified using a dialysis method (MWCO $3.5 \mathrm{kD}$, Millipore) against distilled water. Finally the $p$-HA-PEG-DSPE solution was lyophilized and stored at $-20^{\circ} \mathrm{C}$. The structure of the conjugates was characterized via ${ }^{1} \mathrm{H}-\mathrm{NMR}$ spectroscopy using $\mathrm{CDCl}_{3}$ as a solvent at $25^{\circ} \mathrm{C}$.

\subsection{Preparation and characterization of $p$-HA-PEG- DSPE micelles}

(1) Preparation of micelles. Micelles loaded with coumarin 6 , DiR or DTX were prepared using the thin-film hydration and extrusion method [26]. A mixture of mPEG-DSPE/pHA-PEG-DSPE (95:5 by molar) with coumarin 6 , DiR or DTX in acetonitrile was rotary evaporated to form a thin film. Then the film was vacuum desiccated overnight. To form spontaneous self-assembly of the micelles, the dried polymeric film was dissolved in $10 \mathrm{mmol} / \mathrm{L}$ HEPES-buffered saline (HBS, $\mathrm{pH}$ 7.4) and incubated at a constant temperature of $37^{\circ} \mathrm{C}$ under stirring for $0.5 \mathrm{~h}$. The obtained micelle was filtrated against $0.22 \mu \mathrm{m}$ filter membrane (millipore) to remove unencapsulated DTX. To purify the coumarin 6 and DiR-loaded micelles, size exclusive chromatography was carried on a G-50 gel column using HBS as the mobile phase.

The particle size and distribution were determined via dynamic light scattering (DLS) analysis using Malvern Zetasizer Nano ZS (Malvern, UK) at $25^{\circ} \mathrm{C}$. Each test was measured in triplicate. The morphology of the micelles was 
studied using Transmission Electron Microscope (TEM).

(2) Drug-loading capacity, encapsulation efficiency and in vitro release. To value the encapsulation efficiency (E.E.) and loading capacity (L.C.), the DTX-loaded micelle was diluted in acetonitrile and the concentration of DTX was measured via HPLC (mobile phase: acetonitrile/water $(65 / 35, \mathrm{v} / \mathrm{v}), \lambda: 230 \mathrm{~nm})$ [27]. E.E. was defined as the ratio between actual DTX amount determined and theoretic feeding DTX amount. L.C. was defined as the ratio between DTX amount determined and the weight of lyophilized micelles.

In vitro release profiles of the DTX-loaded micelles were performed in $20 \mathrm{~mL}$ of PBS containing $0.5 \%$ of Tween- 80 . Release samples analyzed via HPLC.

\subsection{Targeting ability study of $p$-HA-PEG-DSPE micelles}

(1) Cellular uptake of $p$-HA-PEG-DSPE micelles. Cellular internalization of coumarin 6-loaded micelles were studied. BCECs cells were seeded into 12-well plates, and each micelle formulation with the same coumarin 6 concentration of $25 \mathrm{ng} / \mathrm{mL}$ was added and incubated at $37^{\circ} \mathrm{C}$ for $1 \mathrm{~h}$. After incubation, the medium was removed and the cells were washed three times with PBS, then visualized using a fluorescent microscope. For quantitative analysis, BCECs cells were trypsinized and centrifuged (1000 r/min, $5 \mathrm{~min})$, washed, resuspended in PBS and analyzed using a flow cytometer (FACSAria, BD, USA).

(2) In vivo distribution of $p$-HA-PEG-DSPE micelles. Potential brain-targeting effect of $p$-HA-PEG-DSPE micelles was evaluated in normal nude mice [25]. Following i.v. administration, time-dependent biodistribution of the DiR-labeled micelles was observed at 1, 2, 4, 8, 12 and $24 \mathrm{~h}$, respectively. The mice were then sacrificed to excise major organs that were photographed ex vivo.

\subsection{Pharmacodynamic study of $p$-HA-PEG-DSPE micelles}

(1) In vitro cytotoxicity assay. In vitro cytotoxicity was determined using the MTT assay [28]. U87 MG cells were seeded to 96 -well plates in $200 \mu \mathrm{L}$ of medium to obtain a concentration of $3 \times 10^{3}$ cells per well, and then the plates were incubated for $24 \mathrm{~h}$. The medium in each well was refreshed with $200 \mu \mathrm{L}$ of the $p$-HA-PEG-DSPE/DTX micelles, mPEG-PEG-DSPE/DTX micelles and Taxotere ${ }^{\circledR}$ respectively and incubated at $37^{\circ} \mathrm{C}$ for $72 \mathrm{~h}$. The concentration of DTX ranged from 0.625 to $102.4 \mu \mathrm{mol} / \mathrm{L}$. Experiments were repeated for at least three times. Percent cell viability was calculated on the basis of optical density values of sample wells versus reference wells (PowerWave XS, Bio-TEK, USA).

(2) Anti-glioblastoma study of $p$-HA-PEG-DSPE/DTX micelles. Therapeutic efficacy of DTX-loaded micelles was investigated in a xenograft mouse model bearing human U87 multiforme glioblastoma [29]. The intracranial U87MG bearing nude mice were randomly divided into four groups $(n=9)$ and treated with the $p$-HA-PEG-DSPE/DTX micelles, unmodified mPEG-PEG-DSPE/DTX micelles, Taxotere ${ }^{\circledR}$ and saline (dose: $8 \mathrm{mg} / \mathrm{kg}$ of body weight at 6,9 , 12 , and $15 \mathrm{~d}$ post-tumor implantation). The survival times were recorded.

\section{Result and discussion}

\subsection{Characterization of $p$-HA-PEG-DSPE}

The conjugation of $p$-HA to PEG-DSPE was confirmed from the ${ }^{1} \mathrm{H}-\mathrm{NMR}$ spectrum. Results showed that the protons of $p$-HA at 6.8-7.8 ppm. The chemical shifts at 3.2-4.0 ppm and 0.8-1.5 represent the PEG (-O- $\left.\mathrm{CH}_{2}-\mathrm{CH}_{2}-\mathrm{O}-\right)$ and the DSPE, respectively. The ${ }^{1} \mathrm{H}-\mathrm{NMR}$ spectrum results indicated the successful synthesis of $p$-HA-PEG-DSPE.

\subsection{Characterization of the $p$-HA-PEG-DSPE micelles}

As shown in Figure 1, the $p$-HA-PEG-DSPE/DTX micelles, the mPEG-DSPE/DTX micelles and blank micelles showed spherical morphology with mean diameters around $18 \mathrm{~nm}$.

The micelles with or without $p$-HA modification showed similar particle sizes, polydispersity indexes as well as drug leakage rates, which indicated that the incorporation of $p$-HA-PEG-DSPE into micelles had no influence on the physical properties of the PEG-DSPE micelle system.

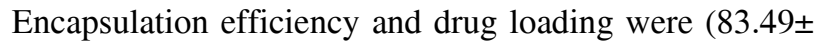
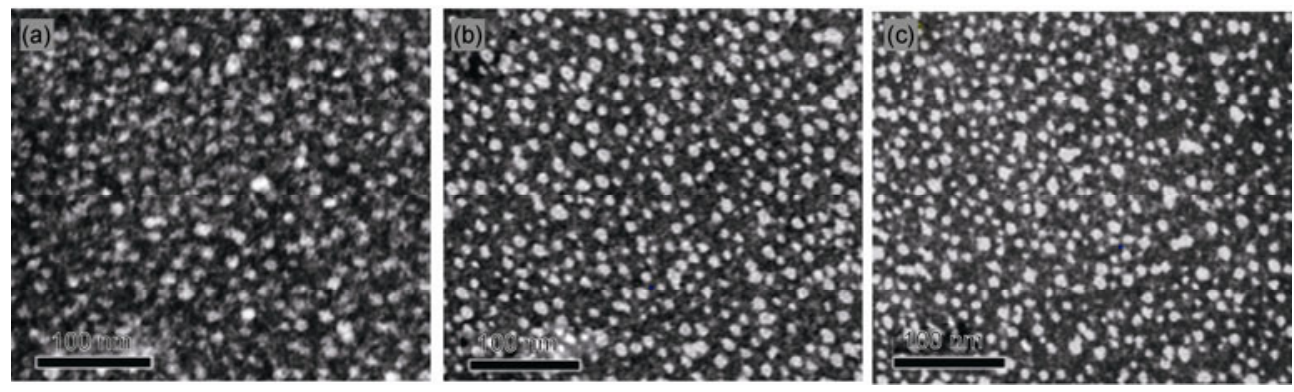

Figure 1 Transmission electron microscope photographs of the micelles (×80000). (a) mPEG-PEG-DSPE/DTX micelles; (b) $p$-HA-PEG-DSPE/DTX micelles; (c) mPEG-PEG-DSPE micelles (blank). 
$1.3) \%,(7.7 \pm 1.2) \%$ for unmodified micelles and $(80.65 \pm$ $1.6) \%$, $(7.47 \pm 1.8) \%$ for $p$-HA-modified micelles, respectively.

Drug leakage rates at $\mathrm{pH} 7.4$ and $\mathrm{pH} 5.0$ in $96 \mathrm{~h}$ are shown in Figure 2. Both of the $p$-HA-PEG-DSPE/DTX micelles and mPEG-PEG-DSPE/DTX micelles exhibited a sustained-release profile in that the total DTX release from micelles was about $40 \%$ in the initial $24 \mathrm{~h}$ in PBS (pH 7.4), $50 \%$ in PBS (pH 7.4) and 74\% in PBS (pH 5.0) for $96 \mathrm{~h}$, respectively (Figure 2(a)). At the same time, there was no obvious difference between $p$-HA-DTX micelle and mPEGDTX micelle (Figure 2(b)).

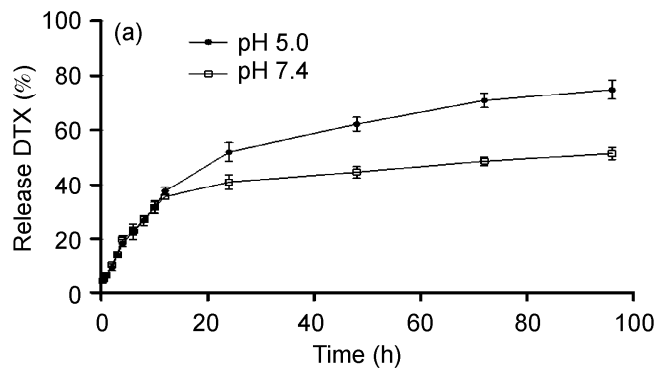

\subsection{Targeting ability study of $p$-HA-PEG-DSPE micelles}

(1) Cellular uptake of the $p$-HA-PEG-DSPE micelles. In vitro cellular uptake was qualitatively determined by fluorescent images and quantitatively as a percentage of coumarin 6 positive cells (Figure 3). As shown in Figure 3, $p$-HAPEG-DSPE/C6 micelles were internalized by BCECs cells more efficiently than mPEG-PEG-DSPE/C6 micelles. Flow cytometry experiments showed the percentage of coumarin 6-positive cells increased from $72.4 \%$ to $92.9 \%$, and mean fluorescence intensity increased from 7.5 to 14.8 . All these

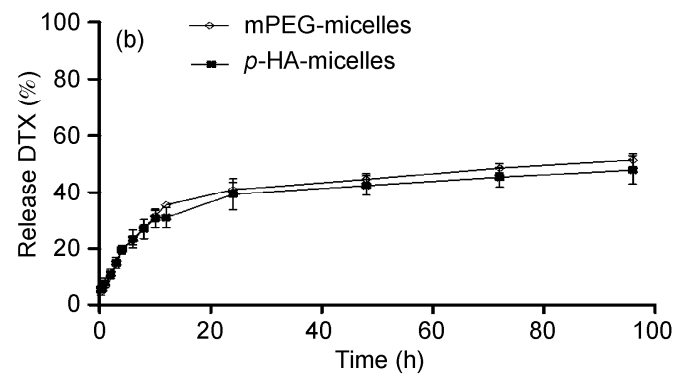

Figure 2 In vitro drug release profiles of DTX-loaded micelles in $\mathrm{pH} 5.0$ and $7.4 \mathrm{PBS}$ at $37^{\circ} \mathrm{C}$ (a) $(n=3$, mean $\pm \mathrm{SD})$; in vitro drug release profiles of DTX-loaded micelles from $7.4 \mathrm{PBS}$ at $37^{\circ} \mathrm{C}$ (b) $(n=3$, mean $\pm \mathrm{SD})$.
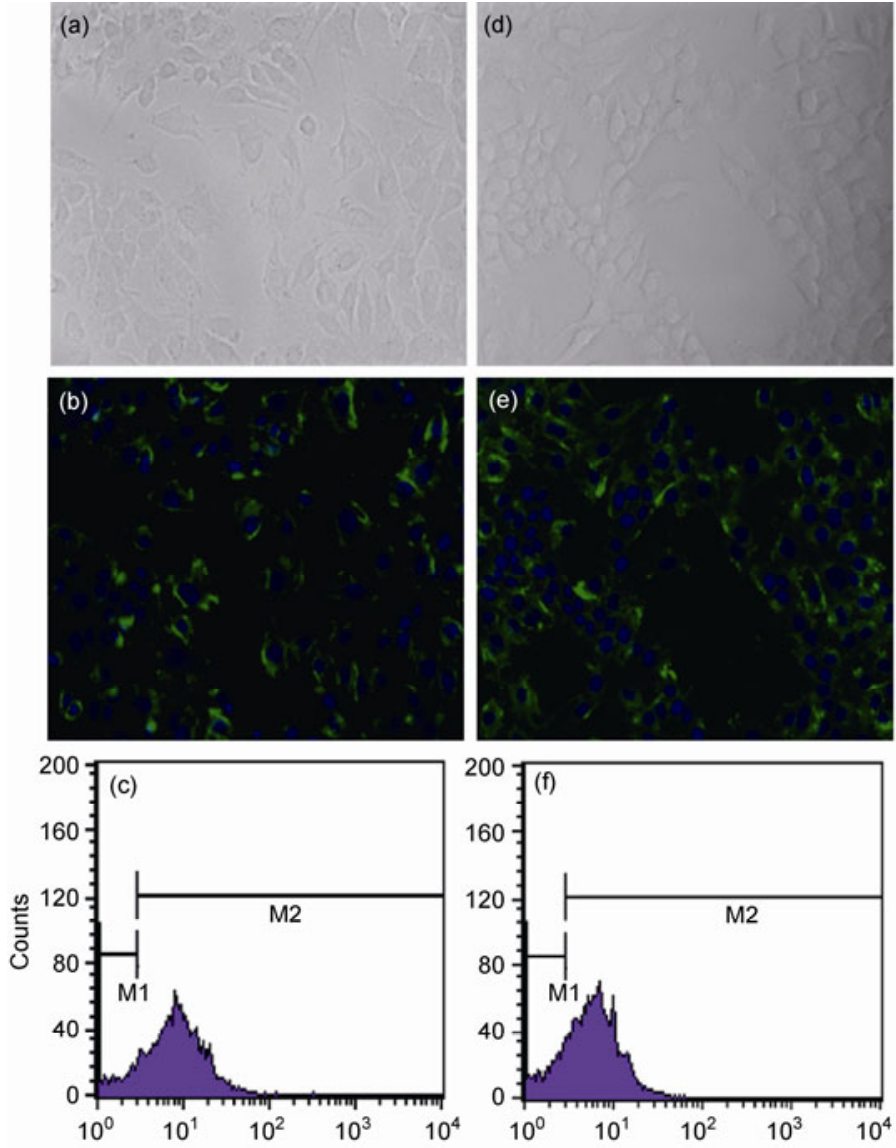
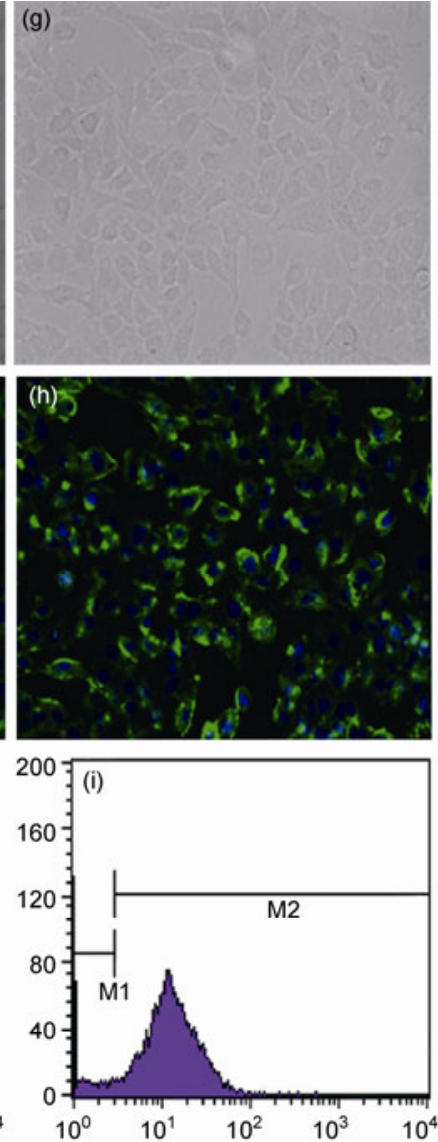

Figure 3 Cellular internalization of free C6 and the C6-loaded micelles. Panels above: micrographs taken in the phase-contrast mode, panels below: the same field viewed in the fluorescence mode. (a)-(c) Free C6; (d)-(f) mPEG-PEG-DSPE/C6; (g)-(i) p-HA-PEG-DSPE/C6. 
results indicated that $p$-HA could enhance micelles uptake by BCECs cells in vitro.

(2) In vivo distribution of $p$-HA-PEG-DSPE/DiR micelles. $p$-HA-PEG-DSPE/DiR micelles and mPEG-DSPE/ $\mathrm{DiR}$ micelles were injected via caudal vein in normal nude mice. Visible fluorescence accumulation appeared much stronger in the brains of $p$-HA-PEG-DSPE/DiR micelle group, compared with the normal micelle group through the entire studies (Figure 4). Ex vivo fluorescent image and semi quantitative of excised organs further confirmed that fluorescence accumulation in the brains treated with the p-HA-PEG-DSPE/DiR micelles were 1.3-1.8 times higher compared with the normal micelle group, except for the highest fluorescence in liver which had high macrophage uptake nature. Compared with the normal micelle group, fluorescence signal in the brains of the $p$-HA-PEG-DSPE micelle group was remarkably stronger at any time post-injection ranged from 2 to $12 \mathrm{~h}$ (Figure 5).

(3) In vitro cytotoxicity studies. In vitro cytotoxicity of DTX-loaded micelles was evaluated on U87 MG cells via MTT assay. Growth inhibition effect against U87 MG cells was observed in both groups. The $\mathrm{IC}_{50}$ values for Taxotere, the mPEG-DSPE/DTX micelles and the $p$-HA-PEG-DSPE/ DTX micelles were $177.8,125.9$, and $104.7 \mathrm{nmol} / \mathrm{L}$, respectively. The $\mathrm{IC}_{50}$ of the DTX-loaded micelles were lower than Taxotere ${ }^{\circledR}$, indicating that the micelles were more efficient to mediate the cytotoxic effect of DTX than Taxotere
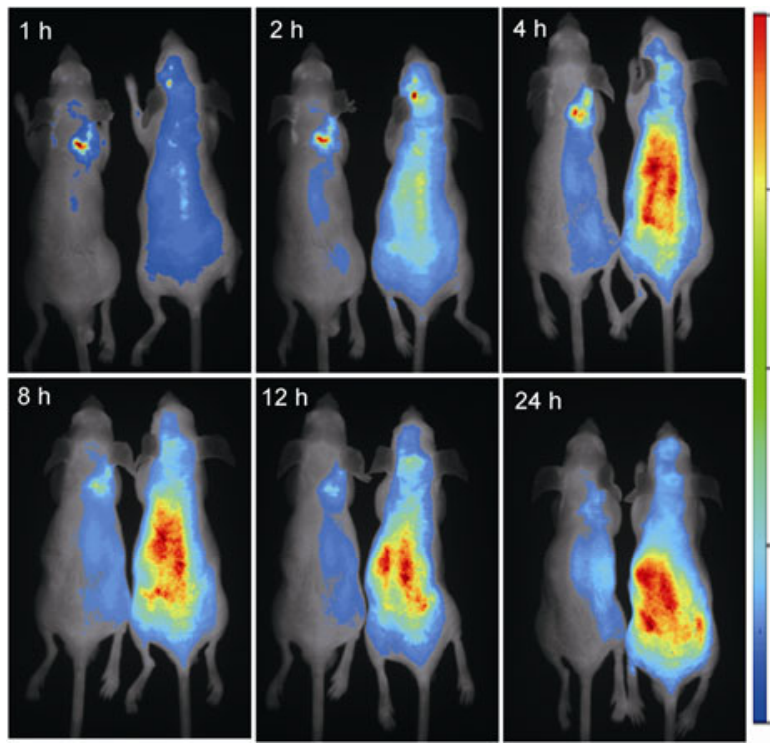

Figure 4 In vivo non-invasive NIR fluorescence images of the $p$-HAPEG-DSPE/DiR (rats at the right side) and the mPEG-DSPE/DiR (rats at the left side) after intravenous administration.

to U87 MG tumor cells.

(4) Anti-glioblastoma studies of the p-HA-PEG-DSPE/ DTX micelles. Figure 6 represents Kaplan-Meier survival curves of the DTX-loaded formulations. The median survival time of the $p$-HA-PEG-DSPE/DTX micelle group, the
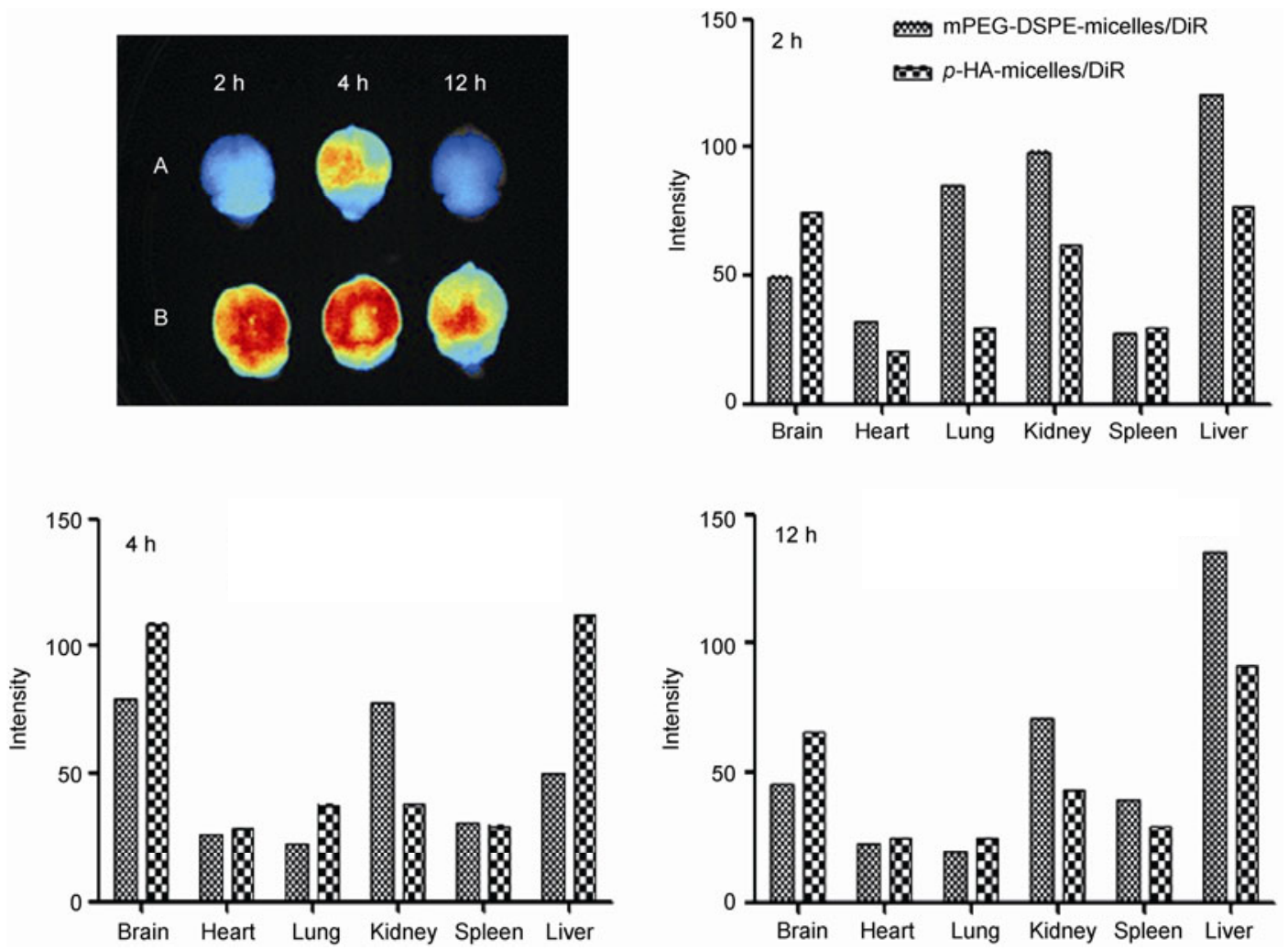

Figure 5 Ex vivo imaging and semi-quantitative analysis of dissected organs at different time points. (A) mPEG-DSPE/DiR micelles; (B) $p$-HA-PEGDSPE/DiR micelles. 


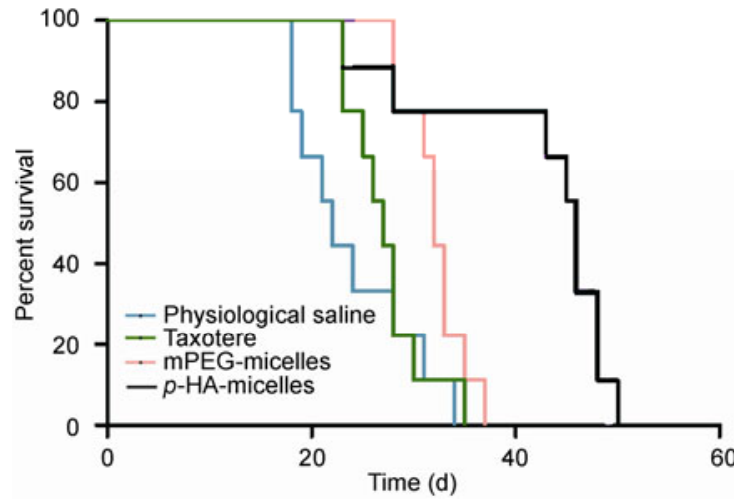

Figure 6 Kaplan-Meier survival curves of mice bearing intracranial U87 glioblastoma.

mPEG-DSPE/DTX micelle group, Taxotere ${ }^{\circledR}$ group and saline group were $45.8,32,27$ and $22 \mathrm{~d}$, repretively. These results demonstrated that the survival of the $p$-HA-PEGDSPE/DTX micelle group was significantly longer than those of other groups $(P<0.05)$. These survival data suggested that $p$-HA could mediate BBB transport of polymeric micelles, thus enhancing therapeutic efficacy of chemotherapy drugs against brain tumors.

\section{Conclusions}

The $p$-HA-modified micelles exhibited strong enhancement on the uptake against the BBB both in vitro and in vivo. All data indicated that this novel small molecular $(p-\mathrm{HA})$ can be used in the anti-tumor drug delivery system which targeting to the brain, and, $p$-HA-conjuncted micelles could have a potential value of clinical application.

This work was supported by the National Basic Research Program of China (2013CB932500), the National Natural Science Foundation of China (81273458) and the Key New Drug Creation Program (2012ZX09304004).

1 Stupp R, Hegi M E, Chakravarti, et al. A chemoradiotherapy in malignant glioma: Standard of care and future directions. J Clin Oncol, 2007, 25: 27-36

2 De Smedt S C, Demeester J, Hennink W E. Cationic polymer based gene delivery systems. Pharm Res, 2000, 17: 113-126

3 Pardridge W M. The blood-brain barrier: Bottleneck in brain drug development. NeuroRx, 2005, 2: 3-14

4 Risau W, Wolburg H. Development of the blood-brain barrier. Trends Neurosci, 1990, 13: 174-178

5 Wang P P, Frazier J, Brem H. Local drug delivery to the brain. Adv Drug Deliv Rev, 2002, 54: 987-1013

6 Béduneau A, Benoit J P. Brain targeting using novel lipid nanovectors. J Control Release, 2008, 126: 44-49

7 Jones A R, Shusta E V. Blood-brain barrier transport of therapeutics via receptor-mediation. Pharm Res, 2007, 24: 1759-1771

8 Huang R Q, Qu Y H, Jiang C, et al. Efficient gene delivery targeted to the brain using a transferrin-conjugated polyethyleneglycol-modified polyamidoamine dendrimer. FASEB J, 2007, 21: 1117-1125

9 Chen Y, Liu L. Modern methods for delivery of drugs across the blood-Brain barrier. Adv Drug Deliv Rev, 2012: 640-655

10 Ulbrich K, Hekmatara T, Herbert E, et al. Transferrin- and transferrinreceptor-antibody-modified nanoparticles enable drug delivery across the blood-brain barrier (BBB). Eur J Pharm Biopharm, 2009, 71: $251-256$

11 Schulingkamp R J, Pagano T C, Hung D, et al. Insulin receptors and insulin action in the brain: Review and clinical implications. Neurosci Amp Biobehav Rev, 2000, 24: 855-872

12 Halatsch ME, Schmidt U, Behnke-Mursch J, et al. Epidermal growth factor receptor inhibition for the treatment of glioblastoma multiforme and other malignant brain tumours. Cancer Treat Rev, 2006, 32: 74-89

13 Lucarelli M, Gennarelli M, Cardelli P, et al. Expression of receptors for native and chemically modified low-density lipoproteins in brain microvessels. FEBS Lett, 1997, 401: 53-58

14 Ngarmukos C, Baur E L, Kumagai A K. Co-localization of GLUT1 and GLUT4 in the blood-brain barrier of the rat ventromedial hypothalamus. Brain Res, 2001, 900: 1-8

15 Robert P H, Allain H. Clinical management of agitation in the elderly with tiapride. Euro Psychiatry, 2001, 1: 42-47

16 Takano A, Suhara T, Okubo Y, et al. The antipsychotic sultopride is overdosed-A PET study of druginduced receptor occupancy in comparison with sulpiride. Int J Neuropsychopharmacol, 2006, 9: 539-545

17 Levey A I, Hersch S M, Ciliax B J, et al. Localization of $\mathrm{D}_{1}$ and $\mathrm{D}_{2}$ dopamine receptors in brain with subtype-specific antibodies. Proc Natl Acad Sci USA, 1993, 90: 8861-8865

18 Saji H D, Tanahashi K K, Yokoyama A, et al. Synthesis, in vitro binding profile and biodistribution of a ${ }^{125}$ I-labeled N-Benzyl pyrrolidinyl benzamide derivative: A potential radioligand for mapping dopamine $\mathrm{D}_{2}$ receptors. Bioorg Med Chem Lett, 2009, 6: 1797-1801

19 Wang T, Petrenko V A, Torchilin V P. Paclitaxel-loaded polymeric micelles modified with MCF-7 cell-specific phage protein: Enhanced binding to target cancer cells and increased cytotoxicity. Mol Pharm, 2010, 7: 1007-1014

20 Shao K, Huang R Q, Jiang C, et al. Angiopep-2 modified PE-PEG based polymeric micelles for amphotericin B delivery targeted to the brain. J Control Release, 2010, 147: 118-126

21 Torchilin V P. Targeted polymeric micelles for delivery of poorly soluble drugs. Cell Mol Life Sci, 2004, 61: 2549-2559

22 Torchilin V P. Targeted pharmaceutical nanocarriers for cancer therapy and imaging. Aaps J, 2007, 9: E128-E147

23 Zhan C Y, Li B, Lu W Y, et al. Micelle-based brain-targeted drug delivery enabled by a nicotine acetylcholine Receptor Ligand. Angew Chem Int Ed, 2011, 50: 5482-5485

24 Liu Y, Lu W Y. Recent advences in brain tumor-targeted nano-drug delivery system. Expert Opin Drug Deliv, 2012, 9: 671-686

25 Li J, Meng Q G, Lu W Y. Benzamide analogue-conjugated polyethylenimine for brain-targeting and gene delivery. J Drug Targeting, 2011, 19: 814-820

26 Han X, Liu J, Lu W Y. 9-NC-loaded folate-conjugated polymer micelles as tumor targeted drug delivery system: Preparation and evaluation in vitro. Int J Pharm, 2009, 372: 125-131

27 Otova B, Va R, Gut I, et al. Effects of paclitaxel, docetaxel and their combinations on subcutaneous lymphomas in inbred Sprague-Dawley/Cub rats. Eur J Pharm Sci, 2006, 29: 442-450

28 Zhan C Y, Gu B, Lu W Y, et al. Cyclic RGD conjugated poly (ethylene glycol)-co-poly(lactic acid) micelle enhances paclitaxel antiglioblastoma effect. J Control Release, 2010, 143: 136-142

29 Yan Z Q, Lu W Y. LyP-1-conjugated PEGylated liposomes: A carrier system for targeted therapy of lymphatic metastatic tumor. J Control Release, 2012, 157: 118-125

Open Access This article is distributed under the terms of the Creative Commons Attribution License which permits any use, distribution, and reproduction in any medium, provided the original author(s) and source are credited. 